\title{
First-principles calculation of the thermoelectric figure of merit for [2,2]paracyclophane-based single-molecule junctions
}

\author{
Marius Bürkle, ${ }^{1, *}$ Thomas J. Hellmuth, ${ }^{2,3}$ Fabian Pauly, ${ }^{3}$ and Yoshihiro Asai ${ }^{1}$ \\ ${ }^{1}$ Nanosystem Research Institute (NRI) 'RICS', National Institute of Advanced Industrial Science and Technology (AIST), \\ Umezono 1-1-1, Tsukuba Central 2, Tsukuba, Ibaraki 305-8568, Japan \\ ${ }^{2}$ Institut für Theoretische Festkörperphysik, Karlsruhe Institute of Technology, 76131 Karlsruhe, Germany \\ ${ }^{3}$ Department of Physics, University of Konstanz, 78457 Konstanz, Germany
}

(Received 30 December 2014; revised manuscript received 27 March 2015; published 20 April 2015)

\begin{abstract}
Here we present a theoretical study of the thermoelectric transport through [2,2]paracyclophane-based singlemolecule junctions. Combining electronic and vibrational structures, obtained from density functional theory (DFT), with nonequilibrium Green's function techniques allows us to treat both electronic and phononic transport properties at a first-principles level. For the electronic part, we include an approximate self-energy correction, based on the DFT $+\Sigma$ approach. This enables us to make a reliable prediction of all linear response transport coefficients entering the thermoelectric figure of merit $Z T$. Paracyclophane derivatives offer a great flexibility in tuning their chemical properties by attaching different functional groups. We show that, for the specific molecule, the functional groups mainly influence the thermopower, allowing us to tune its sign and absolute value. We predict that the functionalization of the bare paracyclophane leads to a largely enhanced electronic contribution $Z_{\mathrm{el}} T$ to the figure of merit. Nevertheless, the high phononic contribution to the thermal conductance strongly suppresses $Z T$. Our work demonstrates the importance to include the phonon thermal conductance for any realistic estimate of the $Z T$ for off-resonant molecular transport junctions. In addition, it shows the possibility of a chemical tuning of the thermoelectric properties for a series of available molecules, leading to equally performing hole- and electron-conducting junctions based on the same molecular framework.
\end{abstract}

DOI: 10.1103/PhysRevB.91.165419

PACS number(s): 63.22.- $\mathrm{m}, 65.80 .-\mathrm{g}$

\section{INTRODUCTION}

Nanostructured materials provide a promising route towards high-performance thermoelectric energy conversion [1-3]. Nanostructuring does not only allow us to improve the thermoelectric performance, but also enables novel applications like on-chip solutions for energy harvesting and refrigeration [2]. Recently it became feasible to probe the thermoelectric properties and energy conversion in molecular junctions [4-6]. Within the different approaches to novel thermoelectric materials, molecular junctions are the ultimate level achievable regarding device miniaturization and provide a great flexibility to tailor their thermoelectric properties, e.g., by chemical synthesis. Presently the applicability of organic thermoelectric materials is limited by the absence of high performance electron-type materials [7]. The possibility to realize equally well performing hole- and electron-type ( $p$ - and $n$-type) molecular junctions and to enhance their efficiency [8-10] makes them very appealing for further scientific investigation. Irrespective of their practical use, they represent ideal systems to study thermoelectric transport at the atomic scale and to improve the understanding of the fundamental processes of nanoscale energy conversion.

On the theory side the combination of DFT-based electronic structure calculations with nonequilibrium Green's function techniques allows an atomistic description of the electric and electronic thermal transport properties. With this approach important trends like the influence of the chemical and structural properties on the charge transport can be successfully captured [4,11-13]. Moreover the recently introduced

*marius.buerkle@aist.go.jp
DFT $+\Sigma$ method, which improves the description of the level alignment at the metal-molecule interface, yields a quantitative agreement with experimental results at low computational costs [14].

Recent computational studies on molecular junctions [15-17] suggest that antiresonances in the electronic transmission close to the Fermi energy can largely enhance the thermopower. The decrease of the electric conductance will be compensated by a simultaneous decrease of the electronic contribution to the thermal conductance. Consequently, this is reported to lead to an increase of the electronic contribution to the thermoelectric figure of merit $Z_{\mathrm{el}} T$. In such situations the overall thermoelectric figure of merit $Z T$ is eventually limited by the phononic contribution to the thermal conductance [see Eqs. (18) and (19) for definitions of $Z_{\mathrm{el}} T$ and $Z T$ ]. In Refs. [15-17], however, this phonon thermal conductance is either neglected or just taken into account in an approximate manner. While it is speculated in Ref. [16] that the phonon thermal conductance might just play a minor role, it is suggested in Ref. [17] that it can have a significant contribution to the overall thermal conductance. Based on first-principles calculations of the relevant transport coefficients, we demonstrate in this paper that it is indispensable for an accurate description of the energy conversion capabilities of molecular junctions to also include the phononic contribution.

As an accurate total energy method, DFT provides the ideal tool to study phonon transport from first principles and has already been applied successfully to various bulk and nanostructured systems [18-20]. However, for molecular junctions first-principles calculations of the phonon thermal conductance have up to now only been performed for a few idealized systems [21-25]. Systematic studies for realistic single-molecule junctions have yet to be provided. 
(a)
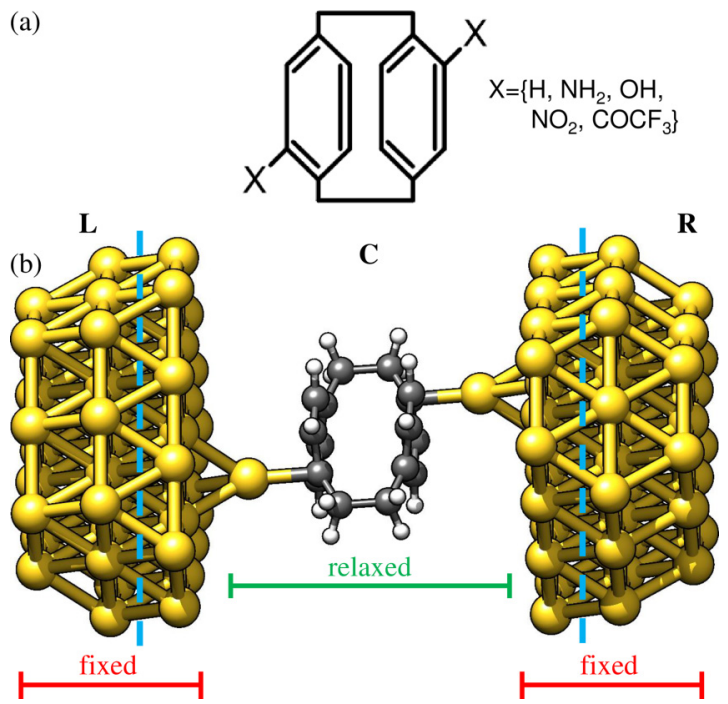

FIG. 1. (Color online) (a) Investigated paracyclophane derivatives with $\mathrm{X}=\left\{\mathrm{H}, \mathrm{NH}_{2}, \mathrm{OH}, \mathrm{NO}_{2}, \mathrm{COCF}_{3}\right\}$ in pseudo-para position. (b) Example of the used contact geometry, here with $\mathrm{X}=\mathrm{H}$.

We present here a fully first-principles-based study of the electron and phonon transport properties for a series of paracyclophane derivatives connected to gold electrodes. We focus on the question of how charge and phonon transport can be tuned chemically by introducing different side groups which are either strongly electron-withdrawing or electron-donating. The chemical structure of the considered [2,2]paracyclophane derivatives is given in Fig. 1(a), namely [2,2]paracyclophane, diamino-[2,2]paracyclophane, dihydroxy-[2,2]paracyclophane, dinitro-[2,2]paracyclophane, and ditrifluoroacetyl-[2,2]paracyclophane, where we, for simplicity, just analyze the pseudo-para isomers. For [2,2]paracyclophane the single-molecule electrical conductance was measured recently [26].

\section{FORMALISM AND METHODS}

\section{A. Electronic, geometric, and vibrational structure}

The electronic structure and the contact geometries are obtained at the DFT level, and the vibrational properties in the framework of density functional perturbation theory (DFPT). Both procedures are implemented in the quantum chemistry software package TURBOMOLE 6.4 [27-29]. We employ the PBE functional [30-33] with empirical dispersion corrections to the total ground-state energy, accounting for long-range van der Waals interactions [34]. As a basis set def2-SV(P) [35] and the respective Coulomb fitting basis [36] are used. To ensure an accurate description of the vibrational properties, we use very tight convergence criteria. Total energies are converged to a precision of better than $10^{-9}$ a.u., while geometry optimizations are performed until the change of the maximum norm of the Cartesian gradient is below $10^{-5}$ a.u..

In the construction of the contact geometries, as shown in Fig. 1(b), we assume the Au atoms of the electrodes in (111) orientation to be fixed at their ideal fcc lattice positions (lattice constant $a_{0}=4.08 \AA$ ). In contrast, the two Au apex atoms and the molecule are fully relaxed. While the precise metal- molecule-metal geometry will influence the thermoelectric properties, we focus here on the effect of the substituents. Due to the time-consuming DFPT calculations, we therefore restrict ourselves to one representative contact configuration. This contact geometry or extended central cluster (ECC) [37] and its division into left $(\mathrm{L})$, central $(\mathrm{C})$, and right $(\mathrm{R})$ regions is shown in Fig. 1(b) for the bare, unsubstituted [2,2]paracyclophane.

\section{B. Electronic transport}

In the Landauer-Büttiker picture the charge current $I$ and the electronic thermal current $Q_{\text {el }}$ are given by [38-40]

$$
\begin{gathered}
I=\frac{2 e}{h} \int d E \tau_{\mathrm{el}}(E)\left[f\left(E, \mu_{\mathrm{L}}, T_{\mathrm{L}}\right)-f\left(E, \mu_{\mathrm{R}}, T_{\mathrm{R}}\right)\right], \quad(1) \\
Q_{\mathrm{el}}=\frac{2}{h} \int d E(E-\mu) \tau_{\mathrm{el}}(E)\left[f\left(E, \mu_{\mathrm{L}}, T_{\mathrm{L}}\right)-f\left(E, \mu_{\mathrm{R}}, T_{\mathrm{R}}\right)\right] .
\end{gathered}
$$

Here, $\tau_{\mathrm{el}}(E)$ is the energy-dependent electron transmission [e.g., from L through C into R, see Fig. 1(b)] and $f(E, \mu, T)=$ $\left\{\exp \left[(E-\mu) / k_{\mathrm{B}} T\right]+1\right\}^{-1}$ is the Fermi function, describing the occupation of the left (right) electron reservoir at the chemical potential $\mu_{\mathrm{L}}\left(\mu_{\mathrm{R}}\right)$ and the temperature $T_{\mathrm{L}}\left(T_{\mathrm{R}}\right)$. The energy of the electrons is measured relative to the average chemical potential $\mu=\left(\mu_{\mathrm{L}}+\mu_{\mathrm{R}}\right) / 2$. This is valid in the linear response regime, assuming $\Delta \mu=\mu_{\mathrm{L}}-\mu_{\mathrm{R}}$ and $\Delta T=T_{\mathrm{L}}-T_{\mathrm{R}}$ to be infinitesimally small.

In linear response the thermoelectric transport coefficients, that is the electrical conductance $G$, thermopower $S$, and electron thermal conductance $\kappa_{\mathrm{el}}$, can be expressed as follows [39,41,42]:

$$
\begin{gathered}
G=\frac{2 e^{2}}{h} K_{0}, \\
S=-\frac{K_{1}}{e T K_{0}}, \\
\kappa_{\mathrm{el}}=\frac{2}{h T}\left(K_{2}-\frac{K_{1}^{2}}{K_{0}}\right) .
\end{gathered}
$$

In the expressions $e=|e|$ is the absolute value of the electron charge, $h$ is the Planck constant, $k_{\mathrm{B}}$ is the Boltzmann constant, and $T=\left(T_{\mathrm{L}}+T_{\mathrm{R}}\right) / 2$ is the average junction temperature. The coefficients in Eqs. (3)-(5) are defined as

$$
K_{n}=\int d E \tau_{\mathrm{el}}(E)\left(-\frac{\partial f(E)}{\partial E}\right)(E-\mu)^{n},
$$

where the chemical potential $\mu \approx E_{\mathrm{F}}$ is approximately given by the Fermi energy $E_{\mathrm{F}}$ of the Au electrodes.

The electronic transmission function is expressed within the Green's function formalism [37,38]. The quasiparticle energies, entering the electronic Green's functions, are approximated by the Kohn-Sham eigenvalues, including a "selfenergy correction." This self-energy correction of the DFT $+\Sigma$ method [14] consists of two parts, one that accounts for the underestimation of the electronic gap of the gas-phase molecule in local and semilocal approximations to DFT, the other one for long-range correlations in a junction that stabilize the charge on the molecule through image charges in the electrodes. Details regarding the self-energy correction can be 
found in Ref. [40]. The electronic contact self energies, on the other hand, are obtained as described in Ref. [37]. To construct the electrode surface Green's function, we use $64 \times 64 k$ points in the transverse direction, which was found to be sufficient to obtain converged electronic transport coefficients.

\section{Phononic transport}

Since phonons are chargeless, they can only carry a heat current. Similar to the electronic expression, this phononic heat current can be calculated in the Landauer-Büttiker picture [43-45] as

$$
Q_{\mathrm{ph}}=\frac{1}{h} \int_{0}^{\infty} d E E \tau_{\mathrm{ph}}(E)\left[n\left(E, T_{\mathrm{L}}\right)-n\left(E, T_{\mathrm{R}}\right)\right] .
$$

The corresponding thermal conductance due to the phonons is given in linear response by

$$
\kappa_{\mathrm{ph}}=\frac{1}{h} \int_{0}^{\infty} d E E \tau_{\mathrm{ph}}(E) \frac{\partial n(E, T)}{\partial T},
$$

where $\tau_{\mathrm{ph}}(E)$ is the phonon transmission and $n(E, T)=$ $\left\{\exp \left(E / k_{\mathrm{B}} T\right)-1\right\}^{-1}$ is the Bose function, characterizing the phonon reservoirs in the left and right electrodes.

Similar to the electronic transmission function, those of the phonons can also be computed within the Green's function formalism [38,44-47]. The system of interest here, namely a molecular junction or more generally a nanoscale conductor, consists of an arbitrary but finite-size scattering region (C) connected to two semi-infinite electrodes ( $\mathrm{L}$ and $\mathrm{R}$ ). For small displacements $\left\{Q_{\xi}\right\}$ around the atomic equilibrium positions $\left\{R_{\xi}^{(0)}\right\}$ the phononic Hamiltonian in the harmonic approximation can be written as

$$
\hat{H}=\frac{1}{2} \sum_{\xi} \hat{p}_{\xi}^{2}+\frac{1}{2 \hbar^{2}} \sum_{\xi \chi} \hat{q}_{\xi} \hat{q}_{\chi} K_{\xi \chi} .
$$

Here we have introduced mass-weighted displacement operators $\hat{q}_{\xi}=\sqrt{M_{\xi}} \hat{Q}_{\xi}$ and mass-scaled momentum operators $\hat{p}_{\xi}=\hat{P}_{\xi} / \sqrt{M_{\xi}}$ as conjugate variables, obeying $\left[\hat{q}_{\xi}, \hat{p}_{\chi}\right]=$ $\mathrm{i} \hbar \delta_{\xi \chi}$ and $\left[\hat{q}_{\xi}, \hat{q}_{\chi}\right]=\left[\hat{p}_{\xi}, \hat{p}_{\chi}\right]=0$, and $\xi=(j, c)$ denotes a Cartesian component $c=x, y, z$ of atom $j$ at position $\vec{R}_{j}=\vec{R}_{j}^{(0)}+\vec{Q}_{j}$. The phononic system is characterized by its dynamical matrix $K_{\xi \chi}=\hbar^{2} \mathcal{H}_{\xi \chi} / \sqrt{M_{\xi} M_{\chi}}$, which is the second-order mass-weighted derivative (or Hessian) of the total ground state energy with respect to the Cartesian atomic coordinates, $\mathcal{H}_{\xi \chi}=\partial_{\xi \chi}^{2} E$. These harmonic force constants are computed within DFPT.

The local displacement basis allows us to partition the dynamical matrix into L, C, and R parts

$$
\boldsymbol{K}=\left(\begin{array}{ccc}
\boldsymbol{K}_{\mathrm{LL}} & \boldsymbol{K}_{\mathrm{LC}} & \mathbf{0} \\
\boldsymbol{K}_{\mathrm{CL}} & \boldsymbol{K}_{\mathrm{CC}} & \boldsymbol{K}_{\mathrm{CR}} \\
\mathbf{0} & \boldsymbol{K}_{\mathrm{RC}} & \boldsymbol{K}_{\mathrm{RR}}
\end{array}\right) .
$$

Under the assumption of vanishing coupling between $\mathrm{L}$ and $\mathrm{R}, \boldsymbol{K}_{\mathrm{RL}}=\boldsymbol{K}_{\mathrm{LR}}^{T}=\mathbf{0}$, the transmission function for ballistic phonons can be written as $[44,47,48]$

$$
\tau_{\mathrm{ph}}(E)=\operatorname{Tr}\left[\boldsymbol{D}_{\mathrm{CC}}^{\mathrm{r}}(E) \boldsymbol{\Lambda}_{\mathrm{L}}(E) \boldsymbol{D}_{\mathrm{CC}}^{\mathrm{a}}(E) \boldsymbol{\Lambda}_{\mathrm{R}}(E)\right],
$$

where we have expressed the phonon transmission function in terms of Green's functions solely defined on C.
We use the general definition of the nonequilibrium phonon Green's function on the Keldysh contour

$$
D_{\xi \chi}\left(\tau, \tau^{\prime}\right)=-i\left\langle T_{C} \hat{S}_{C}\left(\hat{q}_{\xi}(\tau) \hat{q}_{\chi}\left(\tau^{\prime}\right)\right)\right\rangle,
$$

where the displacement operators $\hat{q}_{\xi}(\tau)$ are in the interaction picture, $\hat{S}_{C}=\exp \left[-\mathrm{i} / \hbar \int_{C} d \tau \hat{H}_{i}(\tau)\right]$ is the time evolution operator on the Keldysh contour, and $T_{C}$ is the corresponding time-ordering operator $[46,48,49]$. To calculate the phononic heat current, we need the retarded and advanced Green's functions in energy space. They are obtained by a Fourier transform

$$
D_{\xi \chi}^{\mathrm{r}, \mathrm{a}}(E)=\frac{1}{\hbar} \int_{-\infty}^{\infty} d t D_{\xi \chi}^{\mathrm{r}, \mathrm{a}}(t) \mathrm{e}^{i E t / \hbar}
$$

of the real-time functions, derived from Eq. (12) as [46,48]

$$
\boldsymbol{D}_{\mathrm{CC}}^{\mathrm{r}}(E)=\left[(E+\mathrm{i} \eta)^{2} \mathbf{1}-\boldsymbol{K}_{\mathrm{CC}}-\boldsymbol{\Pi}_{\mathrm{L}}^{\mathrm{r}}(E)-\boldsymbol{\Pi}_{\mathrm{R}}^{\mathrm{r}}(E)\right]^{-1}
$$

with $\boldsymbol{D}_{\mathrm{CC}}^{\mathrm{a}}(E)=\boldsymbol{D}_{\mathrm{CC}}^{\mathrm{r}}(E)^{\dagger}$ and an infinitesimal quantity $\eta>0$. For noninteracting phonons in the electrodes the corresponding contact self energies can be calculated exactly as

$$
\boldsymbol{\Pi}_{Y}^{\mathrm{r}}(E)=\boldsymbol{K}_{\mathrm{C} Y} \boldsymbol{d}_{Y Y}^{\mathrm{r}}(E) \boldsymbol{K}_{Y \mathrm{C}} .
$$

Here, $\boldsymbol{d}_{Y Y}^{\mathrm{r}}(E)$ is the surface Green's function of lead $Y=$ $\{\mathrm{L}, \mathrm{R}\}$. Additionally, we have defined the spectral density of the electrodes as

$$
\boldsymbol{\Lambda}_{Y}(E)=\mathrm{i}\left[\boldsymbol{\Pi}_{Y}^{\mathrm{r}}(E)-\boldsymbol{\Pi}_{Y}^{\mathrm{a}}(E)\right]
$$

with $\Pi_{Y}^{\mathrm{a}}(E)=\Pi_{Y}^{\mathrm{r}}(E)^{\dagger}$.

Similar to our charge quantum transport approach [37] we also employ for the phononic case a cluster-based procedure. We calculate the dynamical matrix $\boldsymbol{K}_{\mathrm{ECC}}$ of a large but finite cluster, consisting of the molecule of interest and parts of the electrodes. Using the partitioning of the ECC, given in Eq. (10) and depicted in Fig. 1(b), we extract $\boldsymbol{K}_{\mathrm{CC}}, \boldsymbol{K}_{\mathrm{LC}}$ and $\boldsymbol{K}_{\mathrm{RC}}$ from $\boldsymbol{K}_{\mathrm{ECC}}$. What remains to be calculated to determine $\tau_{\mathrm{ph}}(E)$ are the surface Green's functions of the electrodes $\boldsymbol{d}_{Y Y}^{\mathrm{r}}(E)$. They are constructed for perfect semi-infinite crystals. This is justified, since $\boldsymbol{d}_{\mathrm{LL}}^{\mathrm{r}}(E)$ and $\boldsymbol{d}_{\mathrm{RR}}^{\mathrm{r}}(E)$ describe $\mathrm{L}$ and $\mathrm{R}$ at the LC and CR interfaces. According to our choice in Fig. 1(b), these are no physical "surfaces," but they correspond to places in the electrodes where the dynamical matrix has approached bulk values $[22,48]$. The dynamical matrix of the Au electrode is derived from those of a spherical cluster, see Fig. 2(a). As in the crystal the atoms of the Au cluster are positioned on an fcc lattice with lattice constant $a_{0}=4.08 \AA$. We exploit that for large enough clusters, the force constants from the central atom to its neighbors will be similar to the bulk. Following the procedure introduced in Ref. [37] for the charge transport, we construct the dynamical matrix of the crystalline electrode via these extracted bulk couplings. From this we compute surface and bulk Green's functions, $\boldsymbol{d}_{Y Y}^{r}(E)$ and $\boldsymbol{d}_{\text {Bulk }}^{r}(E)$, respectively, iteratively by means of the decimation technique [22,37,48,50].

To check the quality of the extracted bulk parameters, we compare the bulk phonon density of states (DOS) with available experimental data. It can be directly obtained from 
(a)
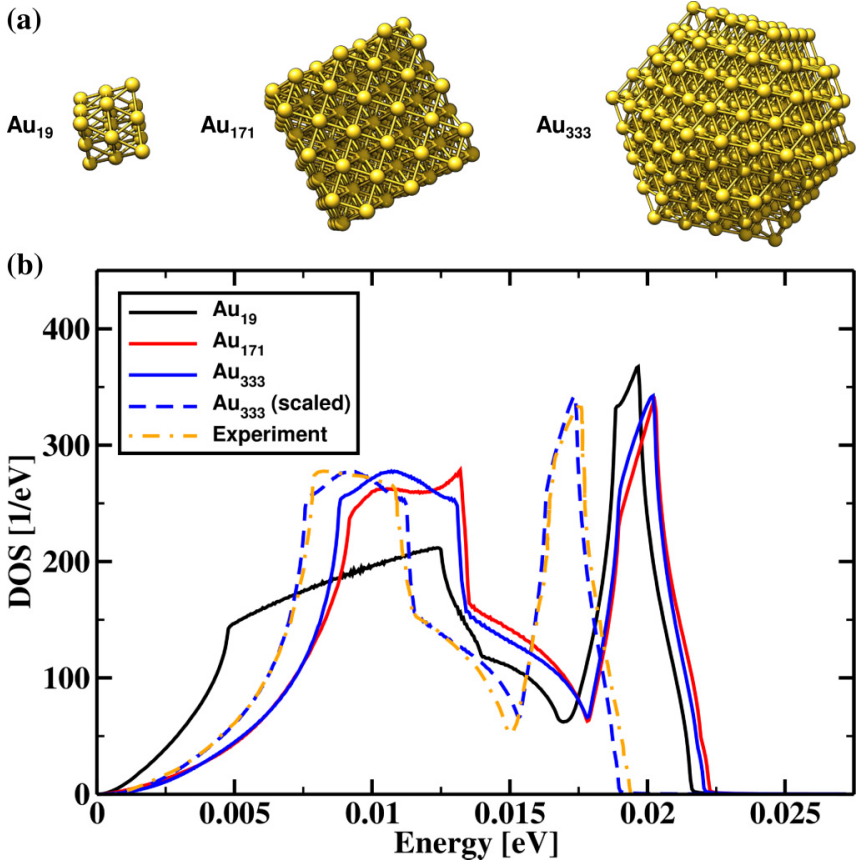

FIG. 2. (Color online) (a) Shape of the three clusters, $\mathrm{Au}_{19}$, $\mathrm{Au}_{171}$, and $\mathrm{Au}_{333}$, used to extract the bulk parameters. (b) Bulk phonon density of states calculated with parameters extracted from the three cluster shown in (a) and experimental DOS from Ref. [51]. The blue dashed curve is the DOS, as obtained from the $\mathrm{Au}_{333}$ cluster, scaled by 0.85 to fit the experimental vibration energies.

the retarded bulk Green's function

$$
\rho(E)=-\frac{2}{\pi} E \operatorname{Im}\left(\operatorname{Tr}\left[d_{\mathrm{Bulk}}^{\mathrm{r}}(E)\right]\right) .
$$

The comparison of the DOS, calculated from parameters extracted from $\mathrm{Au}_{19}, \mathrm{Au}_{171}$, and $\mathrm{Au}_{333}$ clusters, with the experimental data of Ref. [51] is shown in Fig. 2(b). For the smallest cluster $\mathrm{Au}_{19}$ the calculated DOS already reproduces the general features of the experimental one. However, in the lower energy range from 0 to $15 \mathrm{meV}$ the agreement is still poor. Increasing the cluster size to 171 atoms leads to a change especially in the low-energy range of the DOS. If we increase the cluster size further to 333 atoms, we observe only small modifications. Comparing the DOS calculated for the parameters extracted from the $\mathrm{Au}_{333}$ cluster with the experiment [51], we see that the DFT results resemble the experimental DOS closely but overestimate the vibrational energies by around $15 \%$. By scaling the energy axis of the calculated DOS by a factor of 0.85 , we basically obtain a perfect match with the experimental results. Regarding the scaling, we note that in principle both the $x$ and $y$ axes of the DOS plot need to be scaled simultaneously with inverse factors to conserve the energy integral of the DOS. But since the experimental data is measured in arbitrary units, a scaling factor for the $y$ axis is already used.

We want to point out that, in order to obtain a reliable DOS as well as phonon transport properties, it is crucial to choose the broadening factor $\eta$ sufficiently small. Otherwise the sharp features in the spectra are smeared out. Here, we used a broadening of $\eta=6.8 \mu \mathrm{eV}$, for which we obtained both a converged DOS and converged transmission spectra with respect to $\eta$. Due to the small $\eta$ a large number of transverse $k$ points has to be chosen in the decimation procedure. We found the results to be converged for a $k$-point sampling of $512 \times 512$. After the DFPT step, this large number of $k$ points is the bottle neck of the transport calculations. However, the calculation can be parallelized with respect to the $k$ points, and after transforming the surface and bulk Green's functions back to real space, they can be conveniently stored on a hard drive and reused in later calculations.

\section{Thermoelectric figure of merit}

The common measure for thermoelectric efficiency is given by the figure of merit

$$
Z T=\frac{G S^{2}}{\kappa_{\mathrm{el}}+\kappa_{\mathrm{ph}}} T,
$$

which is determined from the thermoelectric transport coefficients in Eqs. (3)-(5) and (8) in the linear response regime. An upper bound for $Z T$ in the limit of vanishing phonon thermal transport $\kappa_{\mathrm{ph}} \rightarrow 0$ is given by the purely electronic contribution

$$
Z_{\mathrm{el}} T=\frac{S^{2} G}{\kappa_{\mathrm{el}}} T=\frac{S^{2}}{L} .
$$

Here, we have introduced the Lorenz number $L=\kappa_{\mathrm{el}} / G T$. With $Z_{\mathrm{el}} T$ we can express the figure of merit in a slightly different form as

$$
Z T=\frac{Z_{\mathrm{el}} T}{1+\kappa_{\mathrm{ph}} / \kappa_{\mathrm{el}}} .
$$

\section{RESULTS AND DISCUSSION}

For the unsubstituted paracyclophane molecule $(X=H)$, $E_{\mathrm{F}}$ is located almost in the middle of the HOMO-LUMO gap (Fig. 3). The conductance is found to be $G_{\mathrm{DFT}+\Sigma}=$ $0.004 G_{0}$ (without self-energy correction $G_{\mathrm{DFT}}=0.07 G_{0}$ ), which compares reasonably well with the experimental data $G_{\text {exp }} \approx 0.01 G_{0}$, given in Ref. [26]. Introducing the substituents leads to a shift of the molecular orbital energies

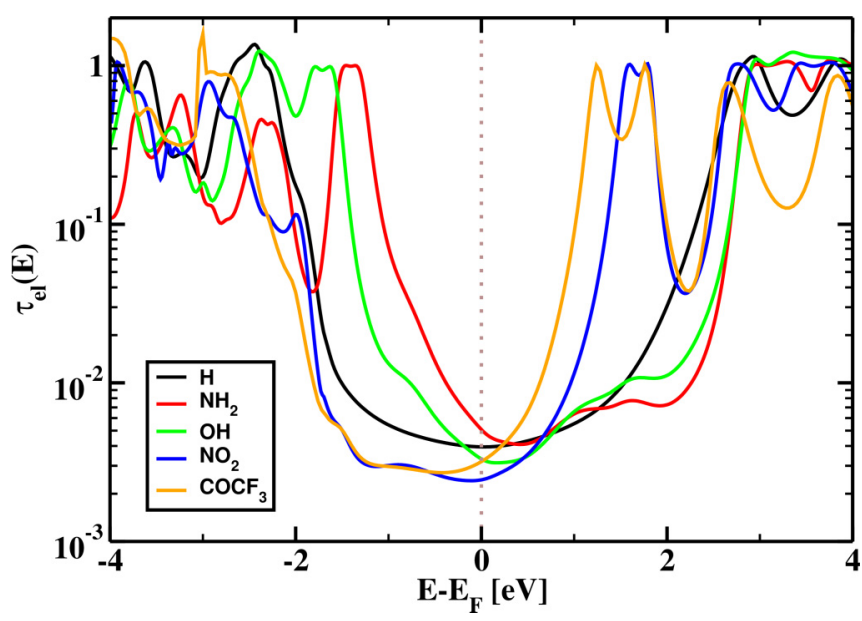

FIG. 3. (Color online) Energy-dependent electronic transmission probability $\tau_{\mathrm{el}}(E)$ relative to $E_{F}$. 

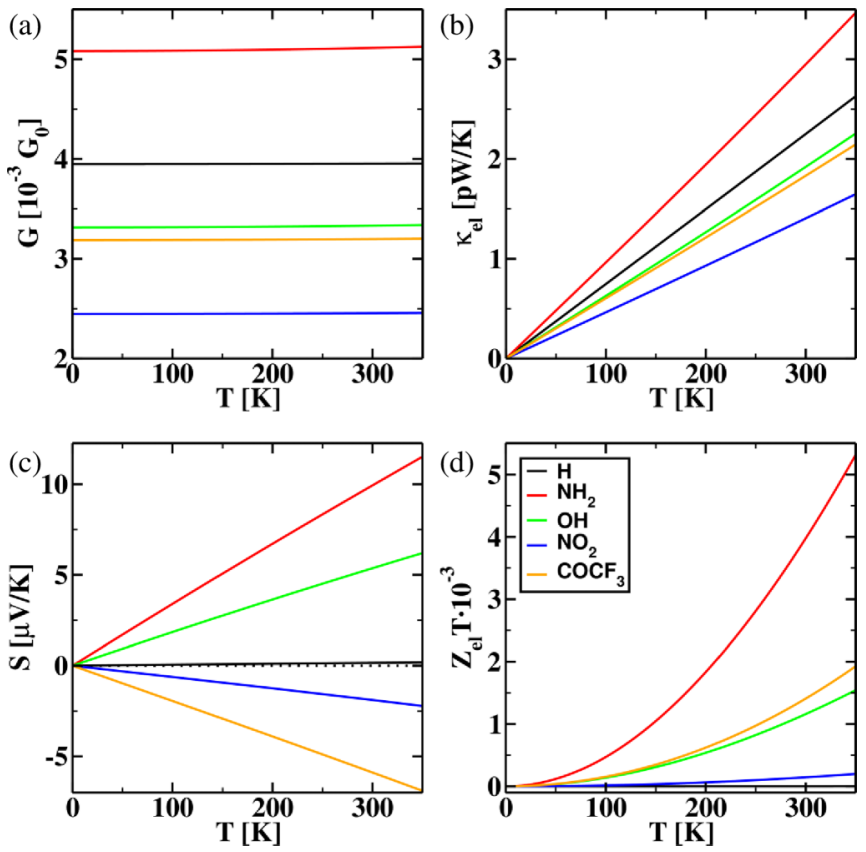

FIG. 4. (Color online) Temperature dependence of the thermoelectric transport coefficients, namely (a) electric conductance, (b) electron thermal conductance, and (c) thermopower. (d) Electronic contribution $Z_{\mathrm{el}} T$ to the figure of merit.

relative to $E_{\mathrm{F}}$ [52]. For the electron-donating substituents $\mathrm{NH}_{2}$ and $\mathrm{OH}$, the molecular frontier orbital energies increase due to the increased Coulomb repulsion in the conjugated $\pi$-electron system of the benzene rings. This moves the HOMO resonance around $1.1 \mathrm{eV}$ closer to $E_{\mathrm{F}}$. The effect is slightly larger for $\mathrm{NH}_{2}$ than for $\mathrm{OH}$ (Fig. 3). The LUMO level is largely unaffected, which suggests that the two substituents mainly couple to the occupied $\pi$ orbitals. For the electronwithdrawing substituents $\mathrm{NO}_{2}$ and $\mathrm{COCF}_{3}$, we observe the opposite effect. The decreased Coulomb repulsion moves the LUMO resonance around $1.5 \mathrm{eV}$ towards $E_{\mathrm{F}}$, with a slightly larger shift for $\mathrm{COCF}_{3}$ (Fig. 3).

In Figs. 4(a)-4(c) the temperature dependence of the thermoelectric transport coefficients is summarized. Before discussing the individual features, it is worth stressing that due to the off-resonant transport and smooth transmission functions around $E_{\mathrm{F}}$, the temperature dependence of the thermoelectric transport coefficients [Eqs. (3)-(5)] is dominated by the lowest order of the Sommerfeld expansion of Eq. (6) $[54,55]$.

Despite the substituent-induced movement of the orbital energies, the transmission at $E_{\mathrm{F}}$ remains roughly constant as compared to the unsubstituted molecule. Hence the influence of the substituents on the electric conductance $G$ as well as the electron thermal conductance $\kappa_{\mathrm{el}}=L T G$ remains small. For $\mathrm{NH}_{2}, G$ and $\kappa_{\mathrm{el}}$ are slightly increased. For the other three substituents a decrease is observed [Figs. 4(a) and 4(b)]. However, the slope of the transmission function at $E_{\mathrm{F}}$, and hence the thermopower $S$, changes substantially. For the unsubstituted molecule the transmission function is flat around $E_{\mathrm{F}}$, resulting in an almost vanishing thermopower [Fig. 4(c)]. On the other hand, for all four substituents the absolute value of $S$ is largely increased [Fig. 4(c)]. Moreover the electron-donating substituents $\mathrm{NH}_{2}$ and $\mathrm{OH}$ tune the slope of $\tau_{\mathrm{el}}(E)$ at $E_{\mathrm{F}}$ to be negative, giving a positive thermopower, characteristic for HOMO-dominated transport ( $p$-type) [5,56-58]. In contrast, the electron-withdrawing substituents give rise to LUMO-dominated transport ( $n$-type), and hence the thermopower is negative [Fig. 4(c)].

Ultimately, we can combine the thermoelectric transport coefficients to the electronic contribution to the figure of merit $Z_{\mathrm{el}} T$, which is displayed in Fig. 4(d). Due to the almost vanishing thermopower of the unsubstituted molecule, $Z_{\mathrm{el}} T$ remains very small. On the other hand, due to the quadratic dependence of $Z_{\mathrm{el}} T$ on $S$, the enhanced thermopower for the substituted molecules increases $Z_{\mathrm{el}} T$ largely. The $\mathrm{NH}_{2}-$ substituted molecule shows the highest $Z_{\mathrm{el}} T$ of around $4 \cdot 10^{-3}$ at room temperature $(T=300 \mathrm{~K})$. Overall $Z_{\mathrm{el}} T$ remains rather small for the [2,2]paracyclophane derivatives studied here. The fact that it is possible to tune the system to be either $p$ or $n$ type by simply replacing the substituents is very encouraging, as this simple approach can be easily transferred to other $\pi$-conducting systems.

Now we turn to the phononic contribution to the thermal conductance, which is often neglected in the calculations or treated in an approximate manner. Here, however, we determine also the phononic thermal conductance accurately from first principles, as described in Sec. II C. Compared to the molecular vibration spectrum [Fig. 5(a)], the electrode phonon density of states (DOS) is finite only in a narrow energy window [Fig. 5(b)]. Even if the presence of the electrodes can
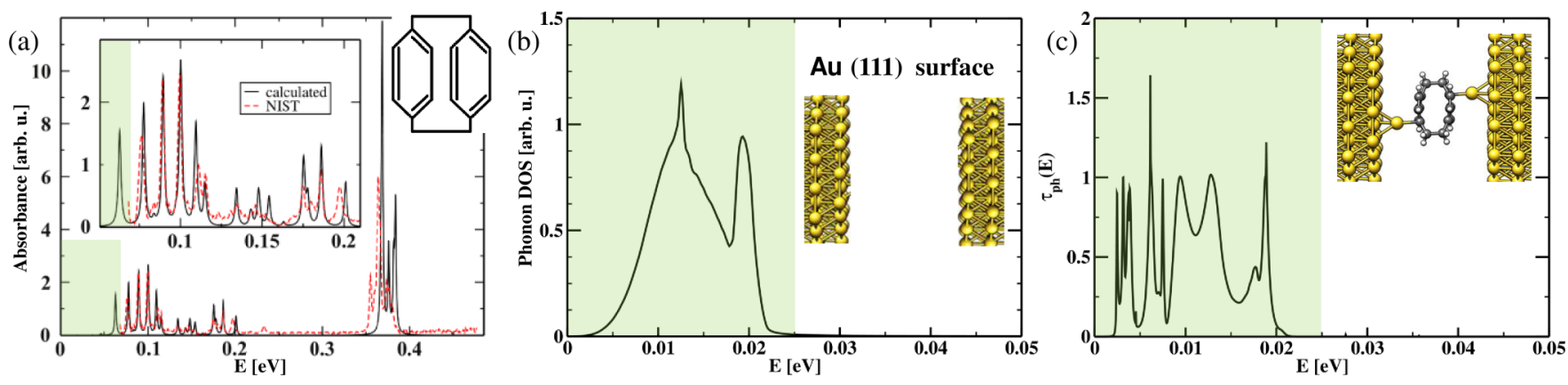

FIG. 5. (Color online) (a) Calculated and measured (Ref. [53]) infrared spectra of [2,2]paracyclophane in the gas phase, showing the infrared-active molecular vibrations. (b) Phonon DOS of a Au (111) surface. (c) Phonon transmission $\tau_{\text {ph }}(E)$ for [2,2]paracyclophane connected to Au electrodes. Green-shaded areas indicate the energy window, where the electrode phonon DOS is finite. 


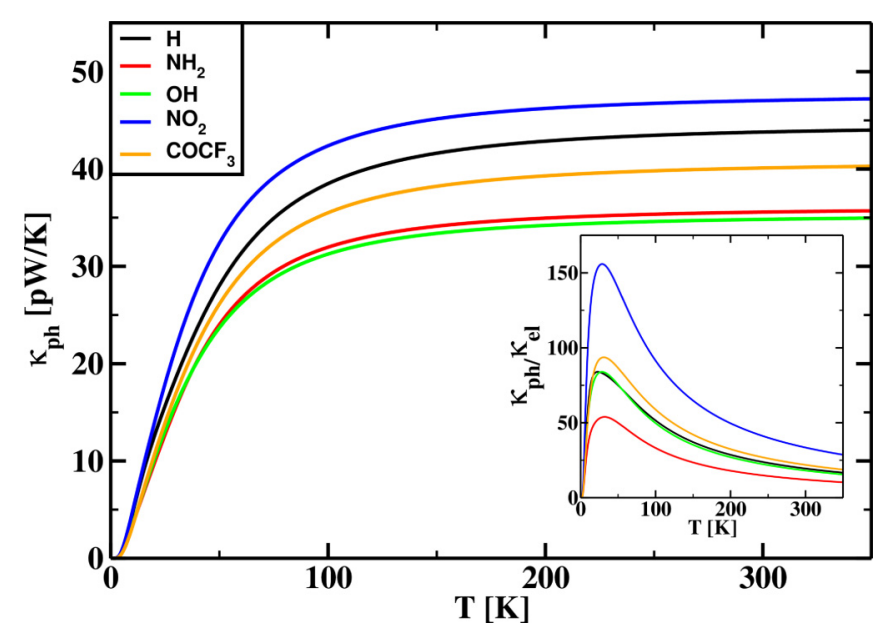

FIG. 6. (Color online) Temperature-dependent phonon thermal conductance. Inset: Ratio $\kappa_{\mathrm{ph}} / \kappa_{\mathrm{el}}$ between phonon and electron thermal conductance.

rather strongly renormalize the molecular vibration spectrum and the character of the individual modes, simply speaking, just those molecular vibrations within the energy window defined by the electrode phonon DOS can contribute to the phonon transport [Fig. 5(c)].

The temperature dependence of the phonon thermal conductance $\kappa_{\mathrm{ph}}$ is displayed in Fig. 6. We obtain the expected ballistic behavior with a steep increase for low temperatures, as the phonon modes are getting occupied, while for higher temperatures $\kappa_{\mathrm{ph}}$ approaches a constant value. Substituting the paracyclophane has a twofold effect. Firstly it influences the molecular vibration spectrum directly, moving modes in and out of the energy window defined by the electrode phonon DOS. Secondly, it changes the binding geometry between the molecule and the $\mathrm{Au}$ electrode, modifying at the same time the coupling between the molecular vibrations and the electrode phonons. Both effects are, however, difficult to separate. Substituting with $\mathrm{NO}_{2}$ increases the phonon thermal

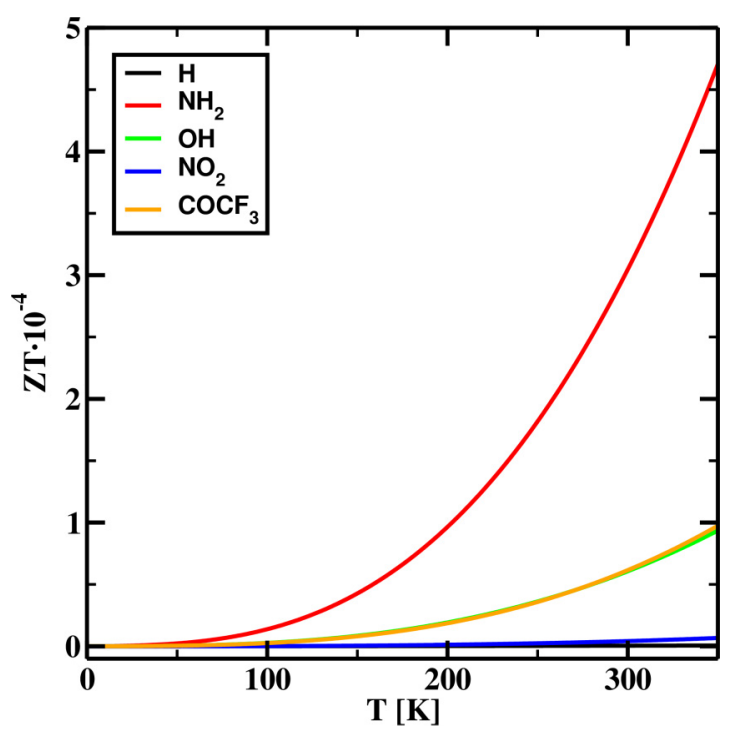

FIG. 7. (Color online) Temperature dependence of the figure of merit $Z T$, including both electronic and phononic linear response transport coefficients. conductance, while the other three substituents decrease it compared to the unsubstituted molecule. Eventually $\kappa_{\mathrm{ph}}$ remains comparable for all five molecules (Fig. 6) and is, in the temperature range $10 \mathrm{~K}<T<400 \mathrm{~K}$, for all molecules at least one order of magnitude larger than the electronic thermal conductance (inset of Fig. 6).

Since $\kappa_{\mathrm{ph}} / \kappa_{\mathrm{el}}>10$ for $10 \mathrm{~K}<T<400 \mathrm{~K}, Z T$ is strongly suppressed according to Eq. (20) (Fig. 7). The largest $Z T$ is obtained for $\mathrm{NH}_{2}$ substitution with $Z T=4 \times 10^{-4}$ at room temperature $(T=300 \mathrm{~K})$. The $\mathrm{OH}$ and $\mathrm{COCF}_{3}$ substituted paracyclophanes have almost the same $Z T$ in the whole temperature range with $Z T=7 \times 10^{-5}$ at $T=300 \mathrm{~K}$, but the former one is hole conducting while the latter one is electron conducting.

\section{CONCLUSIONS}

For a series of paracyclophane-based single-molecule junctions we computed the electrical and heat transport properties fully from first principles. Combining DFT $+\Sigma$ based electronic structure and DFPT-derived harmonic force constants with the Green's function formalism and the Landauer approach allowed us to make quantitative predictions of all linear response transport coefficients relevant for the thermoelectric figure of merit $Z T$.

We showed that the transport properties can be tuned by functionalizing the paracyclophane molecule appropriately. The main influence of the functional groups was found to be on the junction thermopower, where both its absolute value and sign are affected. This is due to the fact that the Fermi energy lies right in the middle of the molecular HOMO-LUMO gap of the bare paracyclophane molecule, leading to a vanishing thermopower and consequently $Z T$. The electron-donating substituents $\mathrm{NH}_{2}$ and $\mathrm{OH}$ tune the thermopower to be positive, while the electron-withdrawing groups $\mathrm{NO}_{2}$ and $\mathrm{COCF}_{3}$ give rise to a negative value. The largest enhancement of the absolute value of the thermopower was found for $\mathrm{NH}_{2}$, followed by $\mathrm{OH}$ and $\mathrm{COCF}_{3}$. The latter two substituents yield a comparable absolute value of $S$ but a different sign.

Naturally, the nonvanishing thermopower increases the electronic contribution $Z_{\mathrm{el}} T$ to the thermoelectric figure of merit largely. However, for the studied off-resonant conduction, the phonon thermal conductance is, even at room temperature, at least one order of magnitude larger than the electronic one, leading to a significant suppression of the overall $Z T$. Therefore we need to stress that it is necessary to include the phonon contribution to the thermal conductance for low-conducting molecular junctions in order to obtain accurate and reliable predictions of $Z T$.

In our present study the largest $Z T$ was found for the $\mathrm{NH}_{2}$-functionalized paracyclophane with $Z T=4 \times 10^{-4}$ at $T=300 \mathrm{~K}$. Even if the $Z T$ remains rather small, we could demonstrate for a series of realistic molecules that it is possible to chemically adjust the transport properties and to enhance the thermoelectric figure of merit as well as to realize $p$ - and $n$-conducting junctions using the same molecular framework.

In the future more complex molecular structures and different functional groups could be used to enhance the thermoelectric figure of merit further. Besides the chemical 
tuning of mainly the electronic transport properties, which was demonstrated in this work, limiting the phonon contribution to the thermal current is necessary to improve $Z T$. Nonlinear effects, leading to increased phonon-phonon scattering, could provide a convenient way to suppress the phonon transport. Such a discussion will be the topic of future work.

\section{ACKNOWLEDGMENTS}

M.B. and Y.A. acknowledge fruitful discussions with $\mathrm{H}$. Nakamura. This work was partly supported by a FY2012 (P12501) Postdoctoral Fellowship for Foreign Researchers from the Japan Society for Promotion of Science (JSPS) and by a JSPS KAKENHI, i.e. "Grant-in-Aid for JSPS Fellows," Grant No. 24-02501. Y.A. is also thankful to another KAKENHI, i.e., Grant-in-Aid for Scientific Research on Innovation Areas "Molecular Architectonics: Orchestration of Single Molecules for Novel Functions" (\#25110009). T.J.H. would like to thank the Karlsruhe House of Young Scientists for financial support during his stay at the Nanosystem Research Institute. F.P. gratefully acknowledges funding from the Carl Zeiss foundation and the Junior Professorship Program of the Ministry of Science, Research, and the Arts BadenWürttemberg.
[1] L. D. Hicks and M. S. Dresselhaus, Phys. Rev. B 47, 16631 (1993).

[2] L. E. Bell, Science 321, 1457 (2008).

[3] M. G. Kanatzidis, Chem. Mater. 22, 648 (2010).

[4] P. Reddy, S.-Y. Jang, R. A. Segalman, and A. Majumdar, Science 315, 1568 (2007).

[5] K. Baheti, J. A. Malen, P. Doak, P. Reddy, S.-Y. Jang, T. D. Tilley, A. Majumdar, and R. A. Segalman, Nano Lett. 8, 715 (2008).

[6] W. Lee, K. Kim, W. Jeong, L. A. Zotti, F. Pauly, J. C. Cuevas, and P. Reddy, Nature (London) 498, 209 (2013).

[7] Q. Zhang, Y. Sun, W. Xu, and D. Zhu, Adv. Mater. 26, 6829 (2014).

[8] S. K. Yee, J. A. Malen, A. Majumdar, and R. A. Segalman, Nano Lett. 11, 4089 (2011).

[9] C. Evangeli, K. Gillemot, E. Leary, M. T. Gonzalez, G. RubioBollinger, C. J. Lambert, and N. Agrait, Nano Lett. 13, 2141 (2013).

[10] J. R. Widawsky, W. Chen, H. Vázquez, T. Kim, R. Breslow, M. S. Hybertsen, and L. Venkataraman, Nano Lett. 13, 2889 (2013).

[11] L. Venkataraman, J. E. Klare, C. Nuckolls, M. S. Hybertsen, and M. L. Steigerwald, Nature (London) 442, 904 (2006).

[12] F. Pauly, J. K. Viljas, and J. C. Cuevas, Phys. Rev. B 78, 035315 (2008).

[13] A. Mishchenko, D. Vonlanthen, V. Meded, M. Bürkle, C. Li, I. V. Pobelov, A. Bagrets, J. K. Viljas, F. Pauly, F. Evers, M. Mayor, and T. Wandlowski, Nano Lett. 10, 156 (2009).

[14] S. Y. Quek, L. Venkataraman, H. J. Choi, S. G. Louie, M. S. Hybertsen, and J. B. Neaton, Nano Lett. 7, 3477 (2007).

[15] C. M. Finch, V. M. García-Suárez, and C. J. Lambert, Phys. Rev. B 79, 033405 (2009).

[16] J. P. Bergfield, M. A. Solis, and C. A. Stafford, ACS Nano 4, 5314 (2010).

[17] R. Stadler and T. Markussen, J. Chem. Phys. 135, 154109 (2011).

[18] A. Ward, D. A. Broido, D. A. Stewart, and G. Deinzer, Phys. Rev. B 80, 125203 (2009).

[19] A. Calzolari, T. Jayasekera, K. W. Kim, and M. B. Nardelli, J. Phys.: Condens. Matter 24, 492204 (2012).

[20] M. N. Luckyanova, J. Garg, K. Esfarjani, A. Jandl, M. T. Bulsara, A. J. Schmidt, A. J. Minnich, S. Chen, M. S. Dresselhaus, Z. Ren, E. A. Fitzgerald, and G. Chen, Science 338, 936 (2012).

[21] D. Nozaki, H. Sevinçli, W. Li, R. Gutiérrez, and G. Cuniberti, Phys. Rev. B 81, 235406 (2010).

[22] N. Sergueev, S. Shin, M. Kaviany, and B. Dunietz, Phys. Rev. B 83, 195415 (2011).
[23] B. K. Nikolić, K. K. Saha, T. Markussen, and K. S. Thygesen, J. Comput. Electron. 11, 78 (2012).

[24] H. Nakamura, T. Ohto, T. Ishida, and Y. Asai, J. Am. Chem. Soc. 135, 16545 (2013).

[25] Y. Asai, J. Phys.: Condens. Matter 25, 155305 (2013).

[26] S. T. Schneebeli, M. Kamenetska, Z. Cheng, R. Skouta, R. A. Friesner, L. Venkataraman, and R. Breslow, J. Am. Chem. Soc. 133, 2136 (2011).

[27] TURBOMOLE 6.4, TURBOMOLE GmbH Karlsruhe, http://www.turbomole.com. TURBOMOLE is a development of University of Karlsruhe and Forschungszentrum Karlsruhe 1989-2007, TURBOMOLE GmbH since 2007.

[28] P. Deglmann, F. Furche, and R. Ahlrichs, Chem. Phys. Lett. 362, 511 (2002).

[29] P. Deglmann, K. May, F. Furche, and R. Ahlrichs, Chem. Phys. Lett. 384, 103 (2004).

[30] P. A. M. Dirac, Proc. R. Soc. A 123, 714 (1929).

[31] J. C. Slater, Phys. Rev. 81, 385 (1951).

[32] J. P. Perdew and Y. Wang, Phys. Rev. B 45, 13244 (1992).

[33] J. P. Perdew, K. Burke, and M. Ernzerhof, Phys. Rev. Lett. 77, 3865 (1996).

[34] S. Grimme, J. Comput. Chem. 27, 1787 (2006).

[35] F. Weigend and R. Ahlrichs, Phys. Chem. Chem. Phys. 7, 3297 (2005).

[36] F. Weigend, Phys. Chem. Chem. Phys. 8, 1057 (2006).

[37] F. Pauly, J. K. Viljas, U. Huniar, M. Häfner, S. Wohlthat, M. Bürkle, J. C. Cuevas, and G. Schön, New J. Phys. 10, 125019 (2008).

[38] S. Datta, Electronic Transport in Mesoscopic Systems (Cambridge University Press, Cambridge, 1997).

[39] U. Sivan and Y. Imry, Phys. Rev. B 33, 551 (1986).

[40] L. A. Zotti, M. Bürkle, F. Pauly, W. Lee, K. Kim, W. Jeong, Y. Asai, P. Reddy, and J. C. Cuevas, New J. Phys. 16, 015004 (2014).

[41] K. Esfarjani, M. Zebarjadi, and Y. Kawazoe, Phys. Rev. B 73, 085406 (2006).

[42] K.-H. Müller, J. Chem. Phys. 129, 044708 (2008).

[43] L. G. C. Rego and G. Kirczenow, Phys. Rev. Lett. 81, 232 (1998).

[44] N. Mingo and L. Yang, Phys. Rev. B 68, 245406 (2003).

[45] T. Yamamoto and K. Watanabe, Phys. Rev. Lett. 96, 255503 (2006).

[46] J.-S. Wang, J. Wang, and N. Zeng, Phys. Rev. B 74, 033408 (2006).

[47] Y. Asai, Phys. Rev. B 78, 045434 (2008).

[48] J.-S. Wang, J. Wang, and J. T. Lü, Eur. Phys. J. B 62, 381 (2008). 
[49] G. Stefanucci and R. van Leeuwen, Nonequilibrium ManyBody Theory of Quantum Systems: A Modern Introduction (Cambridge University Press, Cambridge, 2013).

[50] F. Guinea, C. Tejedor, F. Flores, and E. Louis, Phys. Rev. B 28, 4397 (1983).

[51] J. W. Lynn, H. G. Smith, and R. M. Nicklow, Phys. Rev. B 8, 3493 (1973).

[52] M. Bürkle, L. A. Zotti, J. K. Viljas, D. Vonlanthen, A. Mishchenko, T. Wandlowski, M. Mayor, G. Schön, and F. Pauly, Phys. Rev. B 86, 115304 (2012).

[53] NIST Mass Spec Data Center, S. E. Stein, director, "Infrared Spectra" in NIST Chemistry WebBook, NIST Standard Reference Database Number 69, Eds. P. J. Linstrom and W. G.
Mallard, National Institute of Standards and Technology, Gaithersburg MD, 20899, http://webbook.nist.gov (retrieved November 10, 2014).

[54] N. W. Ashcroft and N. D. Mermin, Solid State Physics (Hartcourt, Orlando, 1976).

[55] G. Gómez-Silva, O. Ávalos-Ovando, M. L. de Guevara, and P. Orellana, J. Appl. Phys. 111, 053704 (2012).

[56] M. Paulsson and S. Datta, Phys. Rev. B 67, 241403(R) (2003).

[57] A. Tan, J. Balachandran, S. Sadat, V. Gavini, B. D. Dunietz, S.-Y. Jang, and P. Reddy, J. Am. Chem. Soc. 133, 8838 (2011).

[58] J. Balachandran, P. Reddy, B. D. Dunietz, and V. Gavini, J. Phys. Chem. Lett. 4, 3825 (2013). 